\section{Limiting shape of profile due to dual-mode fretting wear in contact with an elastomer}

\author{
Xinyu Mao',2, Wei Liu', ${ }^{1,2}$ Yuanzhi $\mathrm{Ni}^{1,2}$ and Valentin L Popov ${ }^{1,3}$
}

Proc IMechE Part C:

Mechanical Engineering Science

2016, Vol. 230(9) 1417-1423

(C) IMechE 2015

Reprints and permissions:

sagepub.co.uk/journalsPermissions.nav DOI: $10.1177 / 0954406215619450$

pic.sagepub.com

\begin{abstract}
We consider fretting wear due to superimposed normal and tangential oscillations of two contacting bodies, one of which is an elastomer with a linear rheology. Similarly to the contact of elastic bodies, at small oscillation amplitudes, the wear occurs only in a circular slip zone at the border of the contact area and the wear profile tends to a limiting form, in which no further wear occurs. It is shown that under assumption of a constant coefficient of friction at the contact interface, the limiting form of the wear profile does depend neither on the particular wear criterion nor on the rheology of the elastomer and can be calculated analytically in a general form. The general calculation procedure and explicit analytic solutions for two initial forms, parabolic and conical, are presented for various combinations of frequencies and phases of normal and tangential oscillations as well as for various linear rheologies of the elastomer.
\end{abstract}

\title{
Keywords
}

Wear, fretting, elastomers, method of dimensionality reduction

Date received: 30 June 20I5; accepted: 4 November 2015

\section{Introduction}

Fretting wear occurs in joints subjected to oscillations with small amplitude. Technically important examples are fretting of tubes in steam generators and heat exchangers, ${ }^{1-3}$ joints in orthopedics, ${ }^{4}$ electrical connectors, ${ }^{5}$ and dovetail blade roots of gas turbines ${ }^{6,7}$ as well as many others. For many applications, contacts between rigid bodies and polymer or elastomer substrates are of interest. Studies on the fretting wear in contacts between polymers and metal counterfaces have shown that small oscillatory contact motions can produce wear of metal surfaces as well as cracking on the polymer surface. ${ }^{8-13}$ The occurrence of either of these processes may depend on the loading conditions (normal load, tangential displacement, and frequency), on the contact geometry, on the material properties and on the environmental conditions. The main wear damage sometimes can be determined using the fretting maps. ${ }^{14}$ In the present paper, we investigate the conditions under which the main damage is due to wear of the "rigid" metallic indenter while the appearing stress distribution is due to deformation and rheology of the polymer.

Depending on particular properties of materials and on loading conditions, fretting wear can lead either to a progressive wear or to some final state in which no further wear occurs. ${ }^{15}$ In the present paper, we consider this latter case and derive the shape of the final, no-wear state.
Consider a rotationally symmetric profile which is brought into contact with a rigid surface and then oscillates in the tangential direction with a given amplitude $u_{x}^{(0)}$ and in the normal direction with the amplitude $u_{z}^{(0)}$. Under assumption of sticking condition, the tangential stress has a singularity at the border of the contact area. ${ }^{16,17}$ This is valid even under consideration of the changing area of contact due to normal oscillation. ${ }^{18}$ This singularity will lead to the appearance of a circular slip zone at the border of the contact area ${ }^{19-21}$ which leads to wear in this zone ${ }^{15}$ while the inner parts of the contact remaining in the state of permanent stick. Note that the existence of the area of permanent stick was shown by Barber et al. $^{22}$ for arbitrary two-dimensional topographies (with not necessarily connected contact area) under very general assumptions and for three-dimensional topographies under usual assumptions of the Cattaneo-Mindlin theory. In the present paper, we consider only axis-symmetrical profiles; however, the

\footnotetext{
'Technische Universität Berlin, Berlin, Germany

${ }^{2}$ Tsinghua University, Beijing, China

${ }^{3}$ National Research Tomsk Polytechnic University, Tomsk, Russia
}

\section{Corresponding author:}

Valentin L Popov, Technische Universität Berlin, Germany. Email: v.popov@tu-berlin.de 
results of the paper ${ }^{22}$ provide a principle possibility of generalization to arbitrary profiles.

In the present paper, we will assume that the friction can be described by a local formulation of the Amonton's law: the surfaces in contact are in the sticking state if tangential stress $\tau$ is smaller than normal pressure $p$ multiplied with a constant coefficient of friction $\mu$, and the tangential stress remains constant after the onset of sliding

$$
\begin{array}{ll}
\tau<\mu p, & \text { stick } \\
\tau=\mu p, & \text { slip }
\end{array}
$$

At the circular border of the stick region with radius $c$, the condition $\tau(c) \leqslant \mu p(c)$ must be fulfilled every moment. Inside this region, the condition $\tau<\mu p$ is valid. Due to wear outside of the sticking region, the local pressure in the sticking region will increase and outside decrease further, independently of whether the experiment is done under conditions of constant normal force or constant indentation depth $d$. This will lead to a progressive wear outside of the region of stick. The wear process will advance until the pressure in the sliding region becomes zero. In this limiting state, the inner parts of the contact will still remain in the sticking state, while the wear rate in the outer parts of the contact tends to zero. The final state of no wear can be considered as a sort of "shakedown" state, in which no further inelastic processes occur. The detailed kinetics of the profile depends on the wear criterion used as well as on the loading conditions (controlled force or controlled indentation). In most cases, the Reye-Archard-Khrushchov wear criterion is used, stating that the wear volume is proportional to the dissipated energy. ${ }^{23-25}$ According to this wear criterion, the wear rate vanishes if either the relative displacement $\Delta u_{x}$ of the bodies or tangential stress in contact is zero. In non-adhesive contacts, the latter means vanishing of the normal pressure $p$. The no-wear condition thus reads

$$
\text { No wear condition : }\left\{\begin{array}{cc}
\text { either } & p=0 \\
\text { or } & \Delta u_{x}=0
\end{array}\right.
$$

From these conditions, it follows that the pressure in the final state is non-zero only inside the stick area and vanishes outside. The whole process and the condition (2) for the final state were formulated in detail in Ciavarella and Hills. ${ }^{15}$ The authors of this paper suggested that it should be possible, using this condition, to deduce the final shape. For the cases of pure tangential oscillations and elastic contact, the shakedown profiles have been calculated analytically in the paper. ${ }^{26}$ In the paper, ${ }^{30}$ the wear process has been simulated and it was confirmed that with increasing number of oscillation cycles the shape of worn profile tends to the limiting form found in Popov. ${ }^{26}$ The same procedure as used in Dimaki et al. ${ }^{27}$ was additionally validated by comparison with finite element simulations in Dimaki et al. ${ }^{28}$ Thus, the approach of the paper ${ }^{26}$ can be considered as validated by at least two independent simulation methods. In the present paper, we will generalize the treatment suggested in the paper $^{26}$ to the dual-mode wear (superposition of normal and tangential oscillations) and the case of visco-elastic contact.

\section{Analytical solution for the final state with the method of dimensionality reduction (MDR)}

To find the final profile, we use the method of dimensionality reduction (MDR). ${ }^{29,30}$ This method allows solving the normal and tangential contact problems for axisymmetric bodies by mapping them to a onedimensional contact of properly defined elastic or viscoelastic foundation. We would like to stress that in spite of analyzing a contact with one-dimensional foundation, the MDR provides exact solutions for the initial three-dimensional contact problem. The corresponding proofs can be found in the literatures ${ }^{29,30}$ as well as in addendums to the monograph. ${ }^{31}$ In Argatov and Popov, ${ }^{32}$ the complete proof of the validity of the MDR in application to viscoleastic indentation was given both for loading and unloading phases of indentation. In the following part, we shortly recapitulate the basics of the method of dimensionality reduction.

The main steps of the MDR are as follows. Given a three-dimensional profile $z=f(r)$, we first determine the equivalent one-dimensional profile, ${ }^{30}$ Figure 1

$$
g(x)=|x| \int_{0}^{|x|} \frac{f^{\prime}(r)}{\sqrt{x^{2}-r^{2}}} \mathrm{~d} r
$$

The back transformation is given by the integral

$$
f(r)=\frac{2}{\pi} \int_{0}^{r} \frac{g(x)}{\sqrt{r^{2}-x^{2}}} \mathrm{~d} x
$$

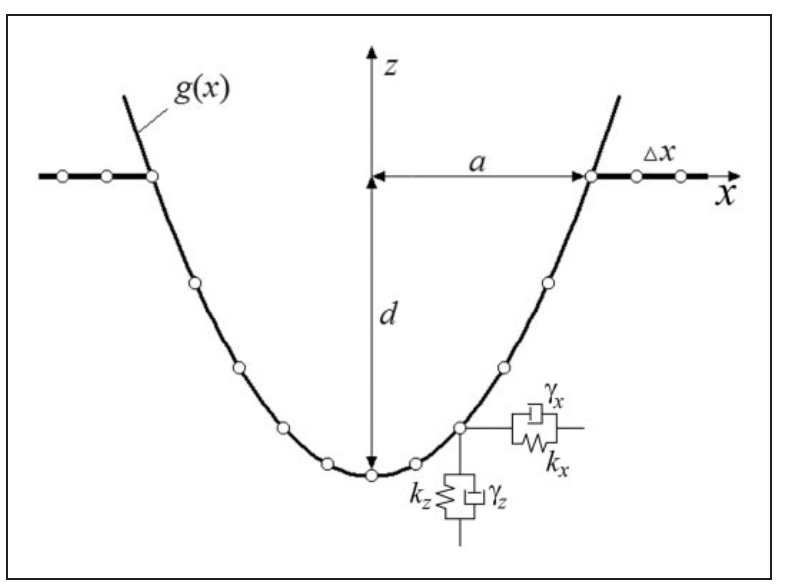

Figure I. Equivalent one-dimensional contact configuration. In this figure, the elements of the viscoelastic foundations are illustrated for the simplest case of Kelvin body (springs and dampers in parallel). 
The profile equation (3) is pressed to a given indentation depth $d$ into an elastic foundation consisting of independent rheological elements. For an incompressible elastomer, the force-displacement relations for each element of the viscoelastic foundation are given by the rules

$$
\begin{aligned}
& \Delta F_{z}=4 \Delta x \int_{-\infty}^{t} G\left(t-t^{\prime}\right) \dot{u}_{z}\left(t^{\prime}\right) \mathrm{d} t^{\prime} \\
& \Delta F_{x}=\frac{8}{3} \Delta x \int_{-\infty}^{t} G\left(t-t^{\prime}\right) \dot{u}_{x}\left(t^{\prime}\right) \mathrm{d} t^{\prime}
\end{aligned}
$$

where $G(t)$ is the time-dependent shear modulus ${ }^{17}$ and $\Delta x$ is the spacing between adjacent elements of the viscoelastic foundations. In particular, in the simplest case of a Kelvin body the elements of the foundation can be represented by spring with stiffness $k_{z}$ and damper $\gamma_{z}$ in the normal direction and stiffness $k_{x}$ and damper $\gamma_{x}$ in the horizontal direction, as shown in Figure 1. The stiffnesses and damping constants are given by the rules

$$
\begin{aligned}
& k_{z}=4 G \Delta x, \quad \gamma_{z}=4 \eta \Delta x, \quad k_{x}=\frac{8}{3} G \Delta x, \\
& \gamma_{x}=\frac{8}{3} \eta \Delta x
\end{aligned}
$$

where $G$ is the shear modulus of the medium and $\eta$ is its viscosity.

The resulting vertical displacements of the springs in the contact region are given by

$$
u_{z}(x)=d-g(x)
$$

It was shown that the one-dimensional profile in the shakedown state has the form ${ }^{18}$

$$
g_{\infty}(x)=\left\{\begin{array}{cc}
g_{0}(x), & \text { for } 0<x<c \\
d, & \text { for } c<x<a
\end{array}\right.
$$

where $c$ is the radius of the region of permanent contact and $g_{0}(x)$ is the one-dimensional MDR-transformed profile corresponding to the initial non-worn profile $f_{0}(r)$. This shape is schematically shown in Figure 2.

The three-dimensional limiting shape can now be calculated by the back transformation equation (4)

$$
f_{\infty}(r)= \begin{cases}f_{0}(r), & \text { for } 0<x<c \\ \frac{2}{\pi} \int_{0}^{c} \frac{g_{0}(x)}{\sqrt{r^{2}-x^{2}}} d x & \text { for } c<x<a \\ +\frac{2}{\pi} d \int_{c}^{r} \frac{1}{\sqrt{r^{2}-x^{2}}} \mathrm{~d} x, & \end{cases}
$$

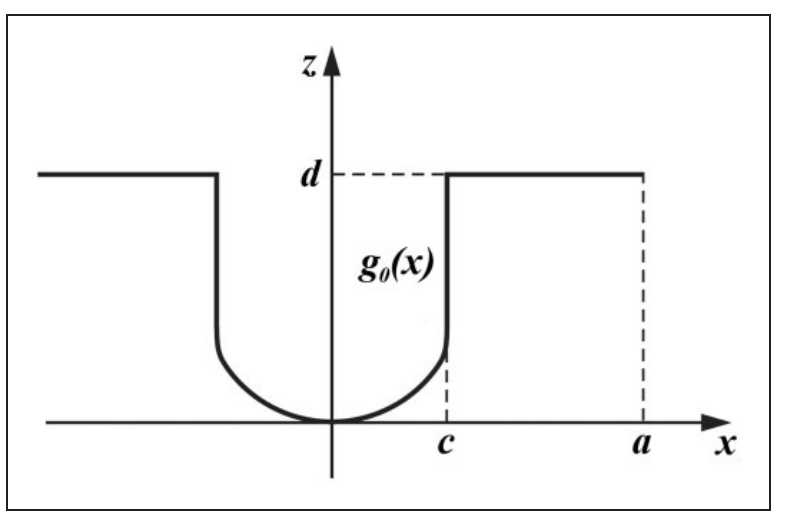

Figure 2. One-dimensional MDR-image of the final "shakedown" profiles.

\section{Limiting shape in the case of dual-mode wear in an elastomer contact}

As stated above, the limiting shape is unambiguously determined by the radius $c$ of the region of permanent stick and the maximum indentation depth during oscillation. Thus, determination of the limiting profile reduced to the determination of the radius $c$ and the $d_{\text {max }}$. Assume that the movement of indenter is given by the equations

$$
d=d_{0}+\Delta d \cos \omega t, \quad u_{x}=u_{x}^{(0)} \cos \left(\omega t+\varphi_{0}\right)
$$

The maximum indentation depth is then determined trivially as

$$
d_{\max }=d_{0}+\Delta d
$$

This value has to be used in equation (10) replacing $d$

$$
f_{\infty}(r)= \begin{cases}f_{0}(r), & \text { for } 0<x<c \\ \frac{2}{\pi} \int_{0}^{c} \frac{g_{0}(x)}{\sqrt{r^{2}-x^{2}}} \mathrm{~d} x & \text { for } c<x<a \\ +\frac{2}{\pi} d_{\max } \int_{c}^{r} \frac{1}{\sqrt{r^{2}-x^{2}}} \mathrm{~d} x, & \end{cases}
$$

Note that the form of the limiting profiles is independent on rheology. The rheology only determines the radius $c$ of the permanent stick region.

Now we are going to determine the radius of the permanent stick. The element at the coordinate $c$ is the last one which is in the permanent contact with the indenter. This means that it follows the movement of the indenter all the time. Displacements of an element at the point $x$ then will be

$$
\begin{aligned}
u_{z}(t) & =d_{0}-g(x)+\Delta d \cos \omega t, \\
u_{x} & =u_{x}^{(0)} \cos \left(\omega t+\varphi_{0}\right)
\end{aligned}
$$


The normal and tangential forces will be given by equations

$$
\begin{aligned}
\Delta F_{z}= & 4 \Delta x G^{\prime}(0)\left(d_{0}-g(x)\right) \\
& +4 \Delta x \Delta d\left(G^{\prime}(\omega) \cos \omega t-G^{\prime \prime}(\omega) \sin \omega t\right) \\
\Delta F_{x}= & \frac{8}{3} \Delta x u_{x}^{(0)}\left(G^{\prime}(\omega) \cos \left(\omega t+\varphi_{0}\right)\right. \\
& \left.-G^{\prime \prime}(\omega) \sin \left(\omega t+\varphi_{0}\right)\right)
\end{aligned}
$$

where $G(\omega)=G^{\prime}(\omega)+i G^{\prime \prime}(\omega)$ is the complex shear modulus, $i$ is imaginary unit, $G^{\prime}(\omega)$ is the storage modulus and $G^{\prime \prime}(\omega)$ is the loss modulus. ${ }^{17}$ The nonslip condition reads $\left|\Delta F_{x}\right| \leqslant \mu \Delta F_{z}$ or

$$
\begin{aligned}
& \frac{2}{3} u_{x}^{(0)}\left|G^{\prime}(\omega) \cos \left(\omega t+\varphi_{0}\right)-G^{\prime \prime}(\omega) \sin \left(\omega t+\varphi_{0}\right)\right| \\
& \leqslant \mu\left[G^{\prime}(0)\left(d_{0}-g(c)\right)+\Delta d\left(G^{\prime}(\omega) \cos \omega t\right.\right. \\
& \left.\left.\quad-G^{\prime \prime}(\omega) \sin \omega t\right)\right]
\end{aligned}
$$

It follows

$$
\begin{aligned}
& g(c) \leqslant d_{0} \\
& -\frac{1}{G^{\prime}(0)}\left[\begin{array}{c}
\frac{1}{\mu} \frac{2}{3} u_{x}^{(0)}\left|\begin{array}{c}
G^{\prime}(\omega) \cos \left(\omega t+\varphi_{0}\right) \\
-G^{\prime \prime}(\omega) \sin \left(\omega t+\varphi_{0}\right)
\end{array}\right| \\
-\Delta d\left(G^{\prime}(\omega) \cos \omega t-G^{\prime \prime}(\omega) \sin \omega t\right)
\end{array}\right]
\end{aligned}
$$

or

$$
\begin{aligned}
g(c)= & \min _{t}\left\{d_{0}-\frac{1}{G^{\prime}(0)}\right. \\
& \left.\times\left[\begin{array}{c}
\frac{1}{\mu} \frac{2}{3} u_{x}^{(0)}\left|\begin{array}{c}
G^{\prime}(\omega) \cos \left(\omega t+\varphi_{0}\right) \\
-G^{\prime \prime}(\omega) \sin \left(\omega t+\varphi_{0}\right)
\end{array}\right| \\
-\Delta d\left(G^{\prime}(\omega) \cos \omega t-G^{\prime \prime}(\omega) \sin \omega t\right)
\end{array}\right]\right\}
\end{aligned}
$$

Equation (19) can be further simplified as

$$
\begin{aligned}
g(c)= & \min _{t}\left\{d_{0}-\frac{|G(\omega)|}{G^{\prime}(0)}\right. \\
& \left.\times\left[\frac{1}{\mu} \frac{2}{3} u_{x}^{(0)}\left|\cos \left(\Omega+\varphi_{0}\right)\right|-\Delta d \cos \Omega\right]\right\}
\end{aligned}
$$

where $\Omega=\omega t+\varphi_{1}$ with $\varphi_{1}$ being the phase angle of the complex shear modulus $G(\omega)$ defined by the equation $\tan \varphi_{1}=G^{\prime \prime}(\omega) / G^{\prime}(\omega)$.

Let us first consider the case when the phase shift between the normal and horizontal oscillation is not fixed (e.g. there is some slow phase creep in the system). In this case, we just have to use the maximum values of $\left|\cos \left(\Omega+\varphi_{1}\right)\right|$ and $\cos \Omega$, and the equation (20) simplifies even further to

$$
g(c)=d_{0}-\frac{|G(\omega)|}{G^{\prime}(0)}\left[\frac{1}{\mu} \frac{2}{3}\left|u_{x}^{(0)}\right|+\Delta d\right]
$$

This equation will be valid also in the case of two different (incommensurate) frequencies of oscillation in normal and tangential directions, in particular in the case when one of the frequencies is much larger than the other one.

However, if the frequencies are equal and phase shift is fixed by some mechanism of mode synchronization, the situation is more complicated. Evaluation of the equation (20) shows that the smallest stick radius (and thus the largest amount of the worn volume) is achieved at $\varphi_{0}=0$ and is given again by the equation (21) while the maximum stick radius (minimum wear volume) is realized at $\varphi_{0}=\pi / 2$

$$
g(c)=d_{0}-\frac{|G(\omega)|}{G^{\prime}(0)} \sqrt{\left(\frac{1}{\mu} \frac{2}{3}\left|u_{x}^{(0)}\right|\right)^{2}+\Delta d^{2}}
$$

For the sake of completeness of analysis, we provide in the following also some exact results in the case of fixed phase difference between oscillations for arbitrary phase shift. In the general case, the finding of the minimum in equation (20) cannot be done just by taking derivative and setting it zero as the function of the right-hand-side of the equation has singular points. The finding of minimum needs therefore analyzing the extrema inside different intervals and comparing them to the values in the singular points.

(a) If $\Omega+\varphi_{0} \in\left(-\frac{\pi}{2} \pm 2 n \pi, \frac{\pi}{2} \pm 2 n \pi\right), n \in Z$, and $\cos \varphi_{0}<\frac{1}{k}$, where

$$
k=\frac{3}{2} \frac{\Delta d}{u_{x}^{(0)}} \mu
$$

then the minimum of the right-hand side of equation (20) inside the interval and the corresponding radius formally calculated with equation (20) is

$$
g\left(c_{1}\right)=d_{0}-\frac{\Delta d}{k} \frac{|G(\omega)|}{G^{\prime}(0)} \sqrt{k^{2}-2 k \cos \varphi_{0}+1}
$$

(b) If $\Omega+\varphi_{0} \in\left(\frac{\pi}{2} \pm 2 n \pi, \frac{3 \pi}{2} \pm 2 n \pi\right), n \in Z$, and $\cos \varphi_{0} \geqslant-\frac{1}{k}$, the minimum inside the interval and the corresponding formally calculated radius of the permanent stick region is given by

$$
g\left(c_{2}\right)=d_{0}-\frac{\Delta d}{k} \frac{|G(\omega)|}{G^{\prime}(0)} \sqrt{k^{2}+2 k \cos \varphi_{0}+1}
$$


(c) If $\Omega+\varphi_{0}=\frac{\pi}{2} \pm 2 n \pi, n \in Z$, then the minimum is achieved in a singular point, the corresponding radius of the permanent stick being

$g\left(c_{3}\right)=d_{0}+\frac{|G(\omega)|}{G^{\prime}(0)} \Delta d \sin \varphi_{0}$

(d) If $\Omega+\varphi_{0}=-\frac{\pi}{2} \pm 2 n \pi, n \in Z$, then, similarly to the case (c)

$$
g\left(c_{4}\right)=d_{0}-\frac{|G(\omega)|}{G^{\prime}(0)} \Delta d \sin \varphi_{0}
$$

We see that the stick radius $c$ is influenced by the relation between $\cos \varphi_{0}$ and $k$. Let us summarize the rules for finding the radius of the permanent stick

1. If $\cos \varphi_{0} \leqslant-\frac{1}{k}\left(\varphi_{0} \in\left(\frac{\pi}{2} \pm 2 n \pi, \frac{3 \pi}{2} \pm 2 n \pi\right), n \in Z\right.$, the same below), $c=\min \left\{c_{1}, c_{3}, c_{4}\right\}=c_{1}$;

2. if $-\frac{1}{k}<\cos \varphi_{0}<\frac{1}{k}, c=\min \left\{c_{1}, c_{2}, c_{3}, c_{4}\right\}$. Then $c=c_{1}$ for $\varphi_{0} \in\left(\frac{\pi}{2} \pm 2 n \pi, \frac{3 \pi}{2} \pm 2 n \pi\right)$;

$c=c_{2}$ for $\varphi_{0} \in\left(-\frac{\pi}{2} \pm 2 n \pi, \frac{\pi}{2} \pm 2 n \pi\right)$;

$c=c_{1}=c_{2}$ for $\varphi_{0}=\frac{\pi}{2} \pm n \pi$; and

3. if $\cos \varphi_{0} \geqslant \frac{1}{k}\left(\varphi_{0} \in\left(-\frac{\pi}{2} \pm 2 n \pi, \frac{\pi}{2} \pm 2 n \pi\right)\right), \quad c=\min$ $\left\{c_{2}, c_{3}, c_{4}\right\}=c_{2}$.

To illustrate complicated non-monotonous and singular behavior of the radius of the region of permanent stick as function of the phase shift $\varphi_{0}$, we calculated $c$ for the case of the parabolic indenter for various parameters $k$ for parameter set $\frac{|G(\omega)|}{G^{\prime}(0)}=1.5$ and $\Delta d / d_{0}=1 / 4$ (see Figure 3 ).

\section{Applications to various shapes and rheologies}

For practical applications of the above rules, first, the effective one-dimensional MDR-profiles in the initial state have to be determined by using equation (3). In the case of a parabolic form of the indenter $f_{0}(r)=r^{2} /(2 R)$, the corresponding MDR-transformed profile is $g_{0}(x)=x^{2} / R$. In the case of a conical profile $f_{0}(r)=r \tan \theta$, the MDR profile is $g_{0}(x)=\frac{\pi}{2}|x| \tan \theta$. The limiting shapes of the profiles are then determined according to equation (13) and are uniquely determined by two parameters: $d_{\max }$ and the radius $c$ of the stick zone. These limiting shapes do not depend on the rheology (which only determines the particular values of the parameters $d_{\max }$ and $c$ ) and coincide with the shapes which were obtained in Popov $^{26}$ for the case of elastic contact. For illustration, we reproduce these shapes in Figure 4.

The above general calculation procedure applies to various linear rheologies of elastomer. Generally, the models contain two fundamental elements, namely linearly elastic springs and dampers. Two of the most common models are Kelvin-Voigt model and the standard rubber model. ${ }^{17}$ The former is a parallel connection of a spring and a damper with spring constants $G$ and $\eta \omega i$, where $G$ is the shear modulus and $\eta$ the dynamic viscosity of the medium. The corresponding complex shear modulus is equal to $\hat{G}(\omega)=G+i \omega \eta$. Thus $|G(\omega)|$ and $G^{\prime}(0)$ are given by

$$
|G(\omega)|=\sqrt{G^{2}+\eta^{2} \omega^{2}} \text { and } G^{\prime}(0)=G
$$

The standard model is the parallel connection of a spring $\left(G_{1}\right)$ and Maxwell element, where Maxwell element is composed of a spring connected in series
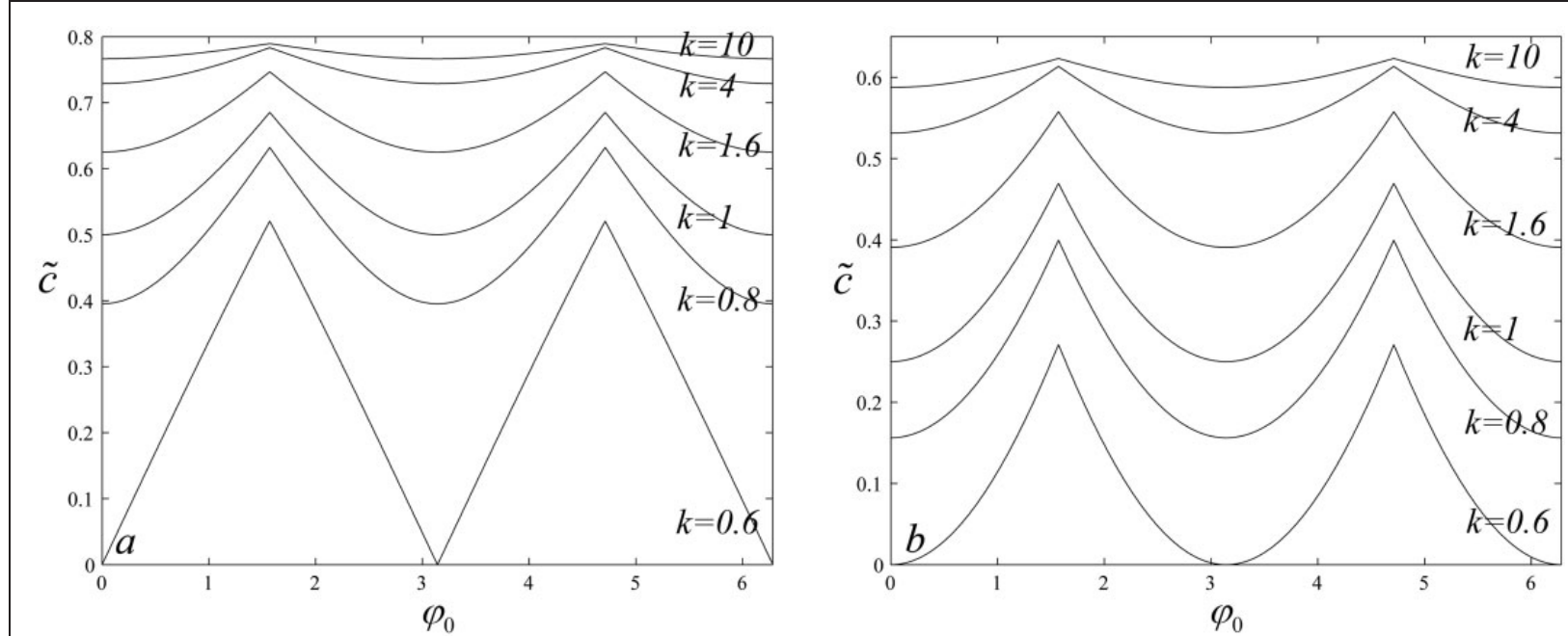

Figure 3. The relation between the normalized radius of permanent stick region $\tilde{c}=c / \sqrt{R d_{0}}$ on $\varphi_{0}$ for the following parameter set: $\frac{|G(\omega)|}{G(0)}=1.5$, and $\Delta d / d_{0}=\mathrm{I} / 4$ for (a) parabolic profile $f_{0}(r)=r^{2} /(2 R)$ and (b) conical profile $f_{0}(r)=r \tan \theta$. 

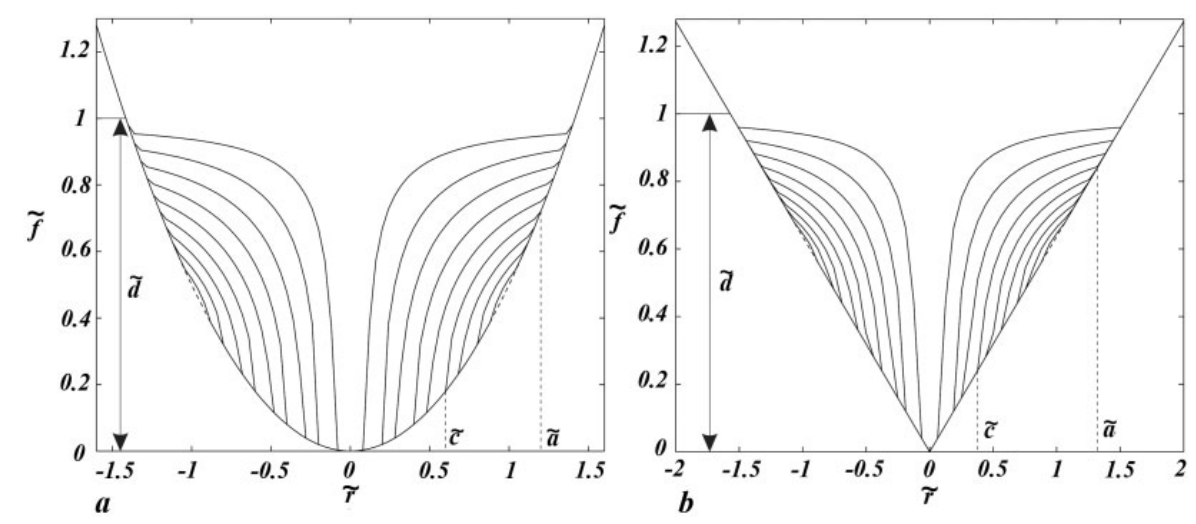

Figure 4. 3D profiles of the worn parabolic (a) and conical (b) profiles in the final state according to equation (I3). Parameters: nine linearly increasing values of $\tilde{c}$ from 0.1 to 0.9 , where $\tilde{d}=d / d_{\max }$ and $\tilde{r}=r / a_{0}$, where $a_{0}$ is initial radius of the contact. Reproduced from Popov. ${ }^{26}$

with a damper $\left(G_{2}, \eta \omega i\right)$. Similarly, it follows

$$
\begin{aligned}
|G(\omega)| & =\frac{1}{\tau^{2} \omega^{2}+1} \sqrt{\left(G_{1}+G_{2}\right)^{2} \tau^{2} \omega^{2}+G_{1}^{2}} \text { and } \\
G^{\prime}(0) & =G_{1}
\end{aligned}
$$

where $\tau=\eta / G_{2}$. With this "complex modulus method" various linear rheologies of elastomer can be applied into the general calculation procedure and explicit analytic solutions.

\section{Various combinations of frequencies of normal and tangential oscillations}

Suppose $\omega_{1}$ and $\omega_{2}$ are the frequencies of normal and tangential oscillations. If $\omega_{1}=\omega_{2}$, the above calculation based on equation (17) is valid. When $\omega_{1} \neq \omega_{2}$, it can happen that the singularities determine the radius of stick region. For arbitrary combination of $\omega_{1}$ and $\omega_{2}$, the calculation is more complicated and is not discussed in the present paper.

\section{Declaration of Conflicting Interests}

The author(s) declared no potential conflicts of interest with respect to the research, authorship, and/or publication of this article.

\section{Funding}

The author(s) disclosed receipt of the following financial support for the research, authorship, and/or publication of this article: This work was partially supported by German Academic Exchange Service.

\section{References}

1. Ko P. Experimental studies of tube frettings in steam generators and heat exchangers. $J$ Pressure Vessel Technol 1979; 101: 125-133.

2. Fisher N, Chow A and Weckwerth M. Experimental fretting-wear studies of steam generator materials. J Pressure Vessel Technol 1995; 117: 312-320.
3. Lee CY, Tian LS, Bae JW, et al. Application of influence function method on the fretting wear of tube-to-plate contact. Tribol Int 2009; 42: 951-957.

4. Collier JP, Mayor MB, Jensen RE, et al. Mechanisms of failure of modular prostheses. Clin Orthopaed Relate Res 1992; 285: 129-139.

5. Antler M. Survey of contact fretting in electrical connectors. IEEE Transact Component Hybrid Manuf Technol 1985; 8: 87-104.

6. Rajasekaran R and Nowell D. Fretting fatigue in dovetail blade roots: experiment and analysis. Tribol Int 2006; 39: 1277-1285.

7. Ciavarella M and Demelio G. A review of analytical aspects of fretting fatigue, with extension to damage parameters, and application to dovetail joints. Int $J$ Solid Struct 2001; 38: 1791-1811.

8. Higham P, Bethune B and Stott F. Changes in the surface morphology of polycarbonate induced by fretting. J Mater Sci 1977; 12: 2503-2510.

9. Higham P, Stott F and Bethune B. Mechanisms of wear of the metal surface during fretting corrosion of steel on polymers. Corros Sci 1978; 18: 3-13.

10. Gaydos P. Fretting wear of polymeric coatings. Wear Mater 1989; 2: 529-535.

11. Kang $\mathrm{C}$ and Eiss N. Fretting wear of polysiloxanepolyimide copolymer coatings as a function of varying humidity. Wear 1992; 158: 29-40.

12. Krichen A, Kharrat $M$ and Chateauminois A. Experimental and numerical investigation of the sliding behaviour in a fretting contact between poly (methylmethacrylate) and a glass counterface. Tribology international 1996; 29: 615-624.

13. Yan FY and Xue QJ. Study of fretting wear behaviors of FEP. J Appl Polym Sci 1998; 67: 1119-1125.

14. Vincent L. Materials and fretting. Fretting fatigue ESIS 1994; 18: 323-337.

15. Ciavarella M and Hills D. Brief note: Some observations on oscillating tangential forces and wear in general plane contacts. Eur J Mech-A/Solid 1999; 18: 491-497.

16. Johnson KL and Johnson KL. Contact mechanics. Cambridge: Cambridge University Press, 1987. 
17. Popov V. Contact mechanics and friction: physical principles and applications. Berlin: Springer Science \& Business Media, 2010.

18. Popov M, Popov V and Pohrt R. Relaxation damping in oscillating contacts. Scientific Report 2015; 5: 16189.

19. Cattaneo C. Sul contatto di due corpi elastici: distribuzione locale degli sforzi. Rend Accad Naz Lincei 1938; 27: $342-348$.

20. Mindlin R. Compliance of elastic bodies in contact. $J$ Appl Mech 2014; 16.

21. Jäger J. Axi-symmetric bodies of equal material in contact under torsion or shift. Archive Appl Mech 1995; 65: 478-487.

22. Barber JR, Davies M and Hills DA. Frictional elastic contact with periodic loading. Int J Solids Struct 2011; 48: 2041-4047.

23. Reye T. Zur Theorie der Zapfenreibung. Der Civilingenieur 1860; 4: 235-255.

24. Khrushchov M and Babichev MA. Investigation of wear of metals. Moscow: Izd-vo AN SSSR, 1960.

25. Archard JF and W Hirst. The Wear of Metals under Unlubricated Conditions. Proceedings of the Royal Society of London. Series A. Mathematical and Physical Sciences 1956; 236(1206): 397-410.

26. Popov VL. Analytic solution for the limiting shape of profiles due to fretting wear. Scientific Report 2014; 4: 3749 .
27. Dimaki AV, Dmitriev AI, Chai YS, et al. Rapid simulation procedure for fretting wear on the basis of the method of dimensionality reduction. Int J Solids Struct 2014; 51: 4215-4220.

28. Dimaki AV, et al. Fast high-resolution simulation of the gross slip wear of axially symmetric contacts. Tribol Transact 2015; DOI: 10.1080/ 10402004.2015.1065529.

29. Popov VL and Heß M. Method of dimensionality reduction in contact mechanics and friction. Berlin: Springer, 2015.

30. Popov VL and Hess M. Method of dimensionality reduction in contact mechanics and friction: A users handbook. I. Axially-symmetric contacts. Facta Universitatis, Series: Mech Eng 2014; 12: 1-14.

31. Popov VL. Kontaktmechanik und Reibung: von der Nanotribologie bis zur Erdbebendynamik. 3rd ed. Berlin: Springer-Verlag, 2015.

32. Argatov II and Popov VL. Rebound indentation problem for a viscoelastic half-space and axisymmetric indenter - Solution by the method of dimensionality reduction. ZAMM. Z Angew Math Mech 2015; 1-12. DOI 10.1002/zamm.201500144. 